\title{
La competencia discursiva potenciada a través de textos multimodales
}

\author{
Mary Luz Hoyos Hoyos \\ Institución Educativa Manuel J. Betancur, Medellín, Colombia \\ maluhoyos@gmail.com \\ Gladys Lucía Acosta Valencia \\ Universidad de Medellín, Medellín, Colombia \\ gacosta@udem.edu.co \\ http://orcid.org/0000-0001-9121-9734 \\ Solbey Morillo Puente \\ Universidad de Medellín, Medellín, Colombia \\ smorillo@udem.edu.co \\ https://orcid.org/0000-0002-2129-1121
}

\section{RESUMEN}

Las habilidades de expresión oral son cruciales para establecer y mantener las relaciones cotidianas del sujeto mediadas por el lenguaje en su interacción con otros y con el contexto mismo en el que se desenvuelve. Esta situación inscribe el discurso oral en la categoría de práctica social. De allí surge el interés del presente artículo, el cual da cuenta de una investigación cuasiexperimental de carácter secuencial con enfoque cuantitativo y mediciones pretest-postest, cuyo propósito central fue analizar la incidencia de una estrategia didáctica con textos multimodales en la potenciación de la competencia discursiva. Los datos fueron examinados desde dos componentes de la estadística:

el descriptivo y el inferencial. Como resultado general del análisis, se determinó que la perspectiva multimodal propició transformaciones favorables en la competencia discursiva de los participantes gracias a que logró articular varias formas de expresión escrita, visual e iconográfica. A través de estas se generaron intercambios verbales entre los estudiantes que activaron procesos discursivos mediante su puesta en escena en el contexto del aula, lo que propició múltiples situaciones de comunicación.

Palabras clave: competencia discursiva; perspectiva dialógica; procedimientos operativos del discurso; textos multimodales; estrategia didáctica.

Cómo citar: Hoyos Hoyos, M. L., Acosta Valencia, G. y Morillo Puente, S. (2020). La competencia discursiva potenciada a través de textos multimodales. Ciencias Sociales y Educación, 9(17), 175-201. https://doi.org/10.22395/ csye.v9n17a9

Recibido: 13 de diciembre de 2019.

Aprobado: 18 de febrero de 2020. 


\section{The Potentiated Discursive Competence Through Multimodal Texts}

\section{ABSTRACT}

Oral expression abilities are crucial for establishing and maintaining the quotidian relation of the subject mediated by language in its interaction with others and in the context itself in which she/ he unfolds. This situation inscribes the oral discourse, which gives an account of quasi-experimental research of sequential character with a quantitative approach and with pretest-protest measuring, which main purpose was analyzing the incidence of a didactic strategy with multimodal texts in the improvement and potentiation of discursive competence. Data was examined from the two components of statistics: the descriptive and the inferential. As a general result of the analysis, the study determined that the multimodal perspective propitiated favorable transformations in the discursive competence of the participants due to the fact that it achieved an articulation of different forms of written, visual and iconographic expression. Via these, verbal exchanges were generated between the students that activated discursive processes through its mise-en-scene in the contexts of the classroom, which propitiated multiple communication situations.

Keywords: discursive competence; dialogical perspective; operative procedures of discourse; multimodal texts; didactic strategic.

\section{A competência discursiva potencializada por meio de textos multimodais RESUMO}

As habilidades de expressão oral são fundamentais para estabelecer e manter as relações cotidianas do sujeito mediadas pela linguagem em sua interação com outros e com o contexto no qual ele se desenvolve. Essa situação coloca o discurso oral na categoria de prática social. A partir disso, surge o interesse deste artigo, o qual evidencia uma pesquisa quase experimental, de caráter sequencial, com abordagem quantitativa e medições pré e pós-teste, cujo objetivo central foi analisar a incidência de uma estratégia didática com textos multimodais na potencialização da competência discursiva. Os dados foram analisados a partir de dois componentes da estatística: o descritivo e o inferencial.
Como resultado geral da análise, foi determinado que a perspectiva multimodal propiciou transformações favoráveis na competência discursiva dos participantes graças à articulação de várias formas de expressão escrita, visual e iconográfica. Por meio destas, foram gerados intercâmbios verbais entre os estudantes que ativaram processos discursivos mediante sua realização no contexto da sala de aula, o que promoveu múltiplas situações de comunicação.

Palavras-chave: competência discursiva; perspectiva dialógica; procedimentos operativos do discurso; textos multimodais; estratégia didática. 


\section{Introducción}

El presente artículo expone los resultados cuantitativos de una mixta que centró su interés en potenciar la competencia discursiva en estudiantes de segundo grado a través de una estrategia didáctica basada en textos multimodales. Esta estrategia surge ante la apremiante necesidad de posicionar la dimensión oral del lenguaje desde los primeros años de escolaridad, dada su relevancia para la democratización de las relaciones sociales, en tanto que, al ser usada cotidianamente por el sujeto, le permite interactuar en un entorno comunicativo. De su uso depende que la participación social sea favorable o, por el contrario, se vea afectada.

En este contexto, la escuela debe ser el lugar comprometido con el individuo para promover la capacidad expresiva del educando, propiciar la circulación de la palabra y favorecer su habilidad de escucha, sin desconocer las nuevas herramientas de interacción social en las que se encuentra inmerso ni las variadas modalidades del lenguaje que responden al fenómeno cultural que lo rodea.

En este sentido, los estudios relacionados con el tema en cuestión —Rodríguez (1995), Núñez (2000), Avendaño (2007), Vásquez (2011), Gutiérrez (2013), Adán (2014) — confirman la relevancia de dirigir la mirada hacia la oralidad en el contexto escolar y darle el lugar que amerita a través de la creación de espacios que la revistan de sentido, de tal manera que el sujeto en formación esté en capacidad de interactuar con su entorno, expresar con apropiación sus opiniones y debatir aquello con lo cual no se identifica. Todos ellos reconocen el discurso oral como una actuación social y proponen como desafío en el ámbito educativo el mejoramiento de la capacidad comunicativa de los educandos.

Con respecto al componente metodológico, se puede decir que es una investigación de campo con enfoque cuantitativo y con un diseño cuasiexperimental con mediciones pretest-postest. Para comprender el problema de estudio, se observó el desarrollo de una actividad diagnóstica y otra posterior a la implementación de los textos multimodales como estrategia didáctica. Cada una de ellas contempló cuatro sesiones de evaluación, a través de las cuales se llevó a cabo un análisis descriptivo e inferencial.

La población objeto de estudio estuvo integrada por un grupo experimental de veintiséis estudiantes de segundo grado, de los cuales se seleccionaron como muestra a ocho participantes a través de un muestreo no probabilístico, intencional, por cuotas. De ellos, cuatro estudiantes (dos niños y dos niñas) sobresalían por su participación oral y por la expresión de sus puntos de vista en torno a los temas abordados en el aula de clase. Por otro lado, cuatro estudiantes (tres niños y una niña) demostraban menor iniciativa para intervenir en los diálogos 
propiciados, pues se les dificultaba expresar sus ideas. Se definió como variable independiente la estrategia didáctica basada en textos multimodales y como variable dependiente la competencia discursiva, la cual está integrada por las dimensiones enunciativa, descriptiva-narrativa y argumentativa.

Como técnica fundamental para la comprensión del fenómeno de estudio, se recurrió a la observación no participante a través de la recolección de datos en una lista de observación integrada por doce ítems distribuidos equitativamente entre las dimensiones antes mencionadas. Este instrumento fue sometido a juicio de expertos para que validaran su contenido (Pedrosa et al., 2013) y se obtuvo como coeficiente de validez de contenido (CVC) 0,93. Esto indica que el instrumento es confiable y demuestra consistencia interna.

Dicha lista de observación fue diligenciada a partir del registro fílmico de cuatro sesiones pretest y cuatro sesiones postest, cada una de ellas con una duración de cincuenta minutos. Para llevar a cabo la calificación, un par de evaluadores fueron entrenados previamente para extraer del registro fílmico la frecuencia de aparición de las conductas que dan cuenta de las tres dimensiones de la competencia discursiva. Se calificó la correlación y se obtuvo un alto grado de acuerdo entre las codificadoras.

El presente artículo se encuentra dividido en tres partes. La primera corresponde al marco conceptual en el cual se mencionan brevemente las investigaciones precedentes sobre el tema de estudio y se desarrollan las categorías centrales asumidas como soporte teórico. En la segunda parte se presentan los resultados obtenidos después de la intervención. Por último, se plantean las conclusiones a las cuales se llegó al terminar la investigación.

\section{Marco conceptual}

\section{Antecedentes}

Los estudios que se enmarcan en el texto multimodal y la competencia discursiva se despliegan con diferentes propósitos y focos de atención: algunos hacen hincapié en su fundamentación conceptual y otros en elementos de orden didáctico. Ambas perspectivas se constituyen en un referente para emprender una indagación en este campo, lo cual permite analizar su posible incidencia en el contexto particular de los participantes. Es relevante resaltar que entre los antecedentes hallados predomina el enfoque cualitativo. A continuación, se hará alusión a ellos como punto de partida para los procesos investigativos de los cuales da cuenta el presente artículo. 
Se encontraron investigaciones como las de Monsalve (2013); Lafontaine y Hébert (2013); Martín y Jiménez (2013); Bravo (2015); Méndez, Urrea y Ayala (2016); Montes (2016); Serrano et al. (2017); Estela y Rebaza (2018) relacionadas con procesos de orden argumentativo, descriptivo, narrativo y de estructuración de diferentes modos de representación. Estos elementos están estrechamente relacionados con la competencia discursiva.

Los autores en mención expresan que ejercitar las acciones enunciadas tiene implicaciones positivas a la hora de favorecer la comunicación, debido a que propician la apertura de espacios de reflexión y la movilización de múltiples estrategias discursivas. Tal como lo evidencian los resultados alrededor del tema enunciado, se produjeron avances entre los sujetos evaluados con respecto a la participación oral, la expresión de ideas y el planteamiento de opiniones soportadas en argumentos. Por otra parte, concluyeron que el fortalecimiento de la competencia discursiva, integrada por componentes verbales y no verbales, se puede lograr cuando el diálogo en el aula es promovido a través de intercambios orales significativos, lo que posibilita el descubrimiento de los diferentes puntos de vista que entran en la conversación, y facilita la argumentación y la combinación del lenguaje con el pensamiento en una dinámica participativa.

Otras investigaciones halladas giran alrededor del texto multimodal, tales como las de Rojas (2013); Oliveira et al. (2014); Arenas y Moreno (2014); Monsalve et al. (2015); Cruz et al. (2016); Chaverra y Gil (2017); Piñeros y Sepúlveda (2017). Sus resultados señalaron que introducir en el ámbito escolar este tipo de textos contribuye a que los estudiantes planteen argumentos plausibles mediante imágenes, vídeos o hipervínculos, y que parafraseen la información para interpretar el texto y ampliar su léxico. Del mismo modo, se propició el reconocimiento de las ideologías presentes en el texto multimodal y se desarrolló la capacidad semiótica a la hora de integrar, comprender y producir mensajes.

Para cerrar, estudios como los de Haquin et al. (2013); Keilwitz y Correa (2014); Melo (2014); Chacón (2015); Gómez (2017); Mendioroz y Asiáin (2017) cobran vigencia para el objeto de estudio debido a que se encuentran relaciones relevantes entre la incorporación de los textos multimodales en el aula de clase y el desarrollo de la competencia discursiva. A su vez, cuestionan la hegemonía del código escrito mediante la utilización de recursos como las imágenes, el simbolismo científico, el vídeo y el sonido.

Los resultados de estas investigaciones señalaron que la interacción generada entre el texto multimodal y los estudiantes condujo al desarrollo de habilidades discursivas, la estimulación del pensamiento divergente, la expresión de ideas, el intercambio de saberes en un contexto real y la emisión de juicios para la construcción colectiva de hipótesis de sentido. 


\section{Perspectiva dialógica del lenguaje}

Los aportes de Bajtin (1982), en cuanto a la concepción dialógica del lenguaje y la teoría del enunciado, se constituyen en un soporte fundamental para abordar más adelante el concepto de competencia discursiva. Este autor concibe el lenguaje como un proceso dialógico en el que intervienen activamente los sujetos involucrados en la práctica discursiva y, a su vez, expresan sus ideas en términos de enunciados que están permeados intrínsecamente por el orden social en el que están inscritos.

En efecto, el oyente, al percibir y comprender el significado lingüístico del discurso, simultáneamente toma, con respecto a este, una activa postura de respuesta: está o no está de acuerdo con el discurso (total o parcialmente), lo completa, lo aplica, se prepara para una acción, etc. Y la postura de respuesta del oyente está en formación a lo largo de todo el proceso de audición y comprensión desde el principio, a veces, a partir de las primeras palabras del hablante.

Toda comprensión de un discurso vivo, de un enunciado viviente tiene un carácter de respuesta (a pesar de que el grado de participación puede ser variado); toda comprensión está preñada de respuesta y de una u otra manera la genera: el oyente se convierte en hablante. (Bajtín, 1982, p. 257)

Lo anterior explica la manera en que se produce la comunicación discursiva, concebida como una práctica verbal de tipo dialogal y de carácter ideológico que surge de la relación intersubjetiva que se produce entre hablante y oyente derivada de la comprensión de los enunciados que se intercambian a través del diálogo. Así pues, en este proceso ambos agentes partícipes juegan un papel importante como actores de expresión de ideas y a la vez contestatarios de los enunciados.

Bajo esta perspectiva, la pregunta por el lenguaje y su vínculo con la competencia discursiva, sumados al papel de estos conceptos en el campo educativo, convocan aspectos de orden filosófico, epistemológico y metodológico que dan razón de una relación entre el desarrollo del lenguaje y la construcción de sujetos discursivos.

\section{La competencia discursiva}

Para comprender la competencia discursiva, se plantea inicialmente la definición de competencia propuesta por Charaudeau (2001), asumida como fundamentación conceptual para los procesos investigativos desarrollados a lo largo del presente artículo. Luego se abordan las competencias del sujeto acogiendo sus postulados con énfasis en la competencia discursiva. Finalmente, se describe lo que tiene que ver con los procedimientos que la rigen. 
En primera instancia, Charaudeau (2001) entiende la competencia como "el resultado de una sabia mezcla de saber-hacer práctico y conocimiento de un área. En lo que al lenguaje se refiere, la competencia abarca conocimientos en torno a datos empíricos, teóricos, creencias, formas y su sentido" (p. 12).

Este concepto recoge los planteamientos de las teorías de la pragmática, la enunciación y la sociolingüística, en tanto que enfoca los hechos del lenguaje como actos orientados hacia la práctica y las circunstancias de comunicación. En adición, vincula el saber al contexto sociocultural, sitúa al sujeto entre sus conocimientos y la manera como los lleva a la práctica en los actos del habla. Es preciso reconocer que dicho saber está condicionado por elementos de su vivencia cotidiana, su ideología, las estructuras del lenguaje y el juicio que suscita en el interlocutor.

De modo más puntual, Charaudeau (2002) afirma que "cada acto de habla es el resultado de la combinación de una situación, una organización discursiva y un cierto uso de las formas" (p. 1). Esto significa que, en este proceso, el sujeto acude a diferentes niveles de construcción de sentido que le permite estructurar sus ideas de acuerdo con el contexto verbal en el que se producen (nivel situacional), manejar un hilo discursivo para hacerse comprender (nivel discursivo) y utilizar herramientas sintácticas que permitan el diálogo fluido (nivel semiolingüístico). Cada uno de estos niveles corresponde precisamente a las competencias del sujeto: el primero corresponde a la competencia comunicacional, el segundo a la competencia discursiva y semántica, y el tercero a la competencia semiolingüística.

Dado que la competencia discursiva es el eje central de la investigación aquí expuesta, es preciso definirla y abordarla de una manera más detallada. En este contexto, Charaudeau (2002) afirma:

La competencia discursiva corresponde al nivel "discursivo" desde el punto de vista del mecanismo de realización del discurso. Representa la capacidad del sujeto para reconocer y manipular los procedimientos operativos de la organización del discurso que se hacen eco de las limitaciones del marco comunicativo. (p. 5)

Bajo esta mirada, la competencia discursiva está relacionada con la capacidad del sujeto para organizar sus ideas, reconocer la información que recibe del otro, comprenderla y discutirla. Por tanto, exige identificar aspectos en el orden de lo social que confluyen en la situación de comunicación, lo que implica la utilización de diferentes estrategias que le permitan un intercambio verbal fluido. Esta competencia corresponde, como se ha precisado, al nivel discursivo desde el punto de vista del mecanismo de realización del discurso, y la competencia semántica, en tanto que los sujetos deben recurrir al conocimiento común para comprender los enunciados que intercambian entre sí en el acto comunicativo. 
Desde este punto de vista, la competencia discursiva cumple una doble función: una función relativa a los modos de organización y del contenido del discurso, y la referente a los tipos de información y sistemas de valores por medio de los cuales los sujetos definen su propia posición.

\section{Procedimientos operativos del discurso}

Los procedimientos operativos de la construcción del discurso se conciben como las acciones que puede incorporar un sujeto en su proyecto comunicativo a la hora de entablar una relación con su interlocutor. A su vez, se constituyen en un modo de organización discursiva que enmarca la situación de comunicación. Estos pueden ser de tres órdenes: enunciativo, descriptivonarrativo o argumentativo.

Los procedimientos enunciativos corresponden a la actitud enunciativa construida por el sujeto hablante, de acuerdo con los datos identificatorios y relacionales de la situación de comunicación, pero también con la imagen que se quiere dar de sí mismo y la imagen que quiere atribuir al otro. (Charaudeau, 2002, p. 3)

Estos procesos hacen alusión al conjunto de actitudes asumidas por el sujeto durante un intercambio verbal, la manera como se expresa, la relación que entabla con el otro hablante y su actuación acorde con las reglas sociales que rigen las prácticas discursivas según el género en el que están emergiendo. En este sentido, las intervenciones de quien habla deben considerar el contexto en el que lo hacen, es decir, analizar si se producen en un ambiente formal o informal.

Por su parte, los procedimientos de orden descriptivo comprenden la manera en que se ponen en juego las habilidades para expresar ideas, el léxico que se emplea y la capacidad para describir acontecimientos y hechos con relación al tema que se aborda. Según Charaudeau (2002),

[l]os procedimientos descriptivo-narrativos consisten tanto en la capacidad de denominar y calificar a los seres del mundo, de forma objetiva y/o subjetiva, para el aspecto descriptivo; como en la capacidad de describir las acciones en el mundo desde el punto de vista de los diversos actores involucrados, para el aspecto narrativo. (p. 4)

Para finalizar, los procedimientos argumentativos consisten en la habilidad con que cuenta un sujeto para explicar, sustentar opiniones, entrar en discusión con el otro para debatir y persuadir a través de ideas consolidadas mediante razonamientos lógicos que pueden llegar a ser creíbles o que inciden en la perspectiva de quien interpreta los enunciados que surgen en una situación de comunicación. En última instancia, son considerados como "la capacidad de organizar cadenas explicativas de causalidad de los eventos y la evidencia de lo que es verdadero, falso o plausible" (Charaudeau, 2002, p. 4). 
En definitiva, es importante reconocer que los procedimientos descritos no son aislados o independientes entre sí. Por el contrario, son usados por el sujeto de manera dinámica y según las necesidades de comunicación que lo motiven a expresarse.

En el ámbito educativo, este enfoque teórico alrededor de la competencia discursiva y los componentes que la integran se constituye en un referente para determinar las acciones discursivas de los sujetos y cobra especial relevancia porque permite ampliar el panorama del aula a posibilidades pedagógicas en las que los estudiantes expresen sus ideas y se vean enfrentados a situaciones que los motiven a polemizar sus puntos de vista. De este modo, se verán convocados a emplear variados procedimientos para potenciar su habilidad discursiva y empoderarse de la palabra como herramienta que le posibilita interactuar con la sociedad de la cual hacen parte y entrar a cuestionar el discurso imperante.

La competencia discursiva permite que el estudiante desarrolle su capacidad para participar activamente en el orden de lo social. En este sentido, lo faculta a la hora de hablar y escuchar. En consecuencia, los sujetos pueden ser capaces de establecer diferencias entre sus puntos de vista y consolidar posiciones críticas frente a las problemáticas que enfrentan a diario.

\section{El texto multimodal}

Para hablar de texto multimodal es preciso hacer alusión, en primera instancia, a la perspectiva multimodal como aquella que acude a múltiples recursos para crear significado. Esto favorece el accionar discursivo en las prácticas pedagógicas y didácticas que se promueven desde la escuela, porque emplea diferentes modos semióticos. Este enfoque ha sido estudiado durante las últimas décadas por autores como Kress y Van Leeuwen (2001); Lemke (1998); Manghi (2013); Oteiza (2009), quienes sentaron sus bases teóricas creando modelos semióticos y discursivos de esta índole, para establecer una definición y caracterización del texto multimodal.

En términos generales, los autores reconocen este tipo de texto como objeto de estudio de la semiótica, disciplina que se encarga de los signos y significaciones que convergen en él. En este sentido, el abordaje multimodal propone que en los objetos textuales siempre se produce un diálogo entre recursos semióticos. En consecuencia, se promueve la interacción entre el sujeto y la producción textual con la que se relaciona.

Dado que vivimos en una sociedad en la que predominan los elementos multimedia, es realmente importante reconocer que a diario interactuamos con este tipo de textos, los cuales surgen como respuesta a las necesidades de comunicación interactiva en la que, además de los elementos lingüísticos, 
se pretende llamar la atención de los seres humanos a través de la imagen y el sonido como valores complementarios del texto.

Un texto multimodal es entendido como el resultado de la acción social, como cualquier instancia de comunicación en cualquier modo o combinación de modos, esto se debe a que las imágenes visuales son producidas, distribuidas y consumidas cultural y socialmente y, por lo mismo, no es posible analizar las imágenes separadas del proceso social en las que ellas existen. (Oteíza, 2009, p. 665)

En este sentido, este tipo de textos utilizados como estrategia didáctica en el contexto escolar supone un desafío porque obliga a mirar más allá de los aspectos lingüísticos para analizar el papel que juegan otros elementos como la imagen y la música. Esta conjugación de modos permite desentrañar varios significados al promover en el aula posibilidades de diálogo en torno a disposiciones discursivas que se han instaurado en el orden de lo social y que hacen parte de la cotidianidad del sujeto en formación, quien, de una manera desprevenida, realiza lectura diaria sin trascender el mero consumo tecnológico. Esta perspectiva se ha hecho ineludible debido a los cambios en las prácticas actuales de lectura y escritura.

En este orden de ideas, es preciso reconocer el texto como "un objeto que representa la materialización de la puesta en escena del acto de lenguaje. Es un resultado siempre singular de un proceso que depende de un sujeto parlante particular y de circunstancias de producción particulares" (Charaudeau, 1983, p. 55). Desde este punto de vista, el texto elevado a la categoría de estrategia didáctica puede llegar a concebirse como un medio que cuenta con unos rasgos que lo caracterizan desde tres aspectos: lo semiótico en clave del cómo decir; las condiciones psicosociales relativas a los tipos de intercambio; y las condiciones en las que se estructuran, entendidas estas como un contrato de comunicación derivado de toda producción del lenguaje.

Desde esta perspectiva, se marca el acento en emplear todos los recursos semióticos a la hora de movilizar interacciones de índole verbal (oral y escrita) y multimodal para construir una representación del lenguaje que permita al estudiante asumir una postura crítica frente al bombardeo tecnológico al que se enfrenta a diario. El hecho de incorporar al interior del aula prácticas que se inscriben en el enfoque multimodal permite que el sujeto reconozca los diferentes símbolos con los que se relaciona y comprenda el valor que adquieren en la sociedad que los encarna.

\section{Estrategia didáctica basada en textos multimodales}

Desarrollar la competencia discursiva durante los grados iniciales de escolarización mediante una estrategia didáctica, reviste un proceso complejo que 
requiere de la conjugación de múltiples elementos, entre los cuales se encuentran el contexto en el que interactúan los estudiantes, la mediación del docente y los recursos didácticos que se empleen para tal propósito. Para efectos de la investigación efectuada, se entiende por estrategia didáctica

[e]l conjunto de procedimientos (métodos, técnicas, actividades) por los cuales el docente y los estudiantes, organizan las acciones de manera consciente para construir y lograr metas previstas e imprevistas en el proceso enseñanza y aprendizaje, adaptándose a las necesidades de los participantes de manera significativa. (Feo, 2010, p. 222)

Para que estos procedimientos puedan ser aplicados en el aula, es necesario que el docente establezca una planificación clara y reflexiva en la que considere un abanico de posibilidades que le permita implementar diferentes acciones para favorecer los procesos de aprendizaje. Estas deben estar entrelazadas bajo la premisa de que el estudiante es un agente activo que adapta y comprende la información a partir de sus expectativas y sus conocimientos previos.

En esta línea de sentido, la estrategia didáctica basada en textos multimodales parte del establecimiento de relaciones dialógicas que se expresan, según Bajtín (1982), como "relaciones de sentido entre toda clase de enunciados en la comunicación discursiva" (p. 309). Estas relaciones son derivadas del intercambio verbal que se propicia de manera intersubjetiva entre los actores involucrados, lo que pone en juego todos los componentes que integran el texto en mención como un dinamizador de los procesos discursivos en el aula.

En consecuencia, cada texto multimodal se convierte en un recurso que posibilita poner en diálogo el contenido y el formato a través de la activación de diversas habilidades discursivas. De esta manera, se logra el tránsito de un objeto textual a su conversión en una estrategia para fortalecer múltiples habilidades discursivas de los sujetos participantes.

\section{Estructura de la estrategia didáctica}

A través del diseño de un conjunto de dieciocho sesiones de intervención pedagógica con una duración de dos horas cada una, se utilizaron los textos multimodales y se implementaron acciones didácticas como el diálogo, el debate, la socialización, la exposición oral y el trabajo colaborativo con el propósito de potenciar las habilidades discursivas orales y generar procesos de interpretación y comprensión en los cuales los estudiantes ponían en juego los procedimientos operativos del discurso (enunciativo, descriptivo-narrativo y argumentativo).

Fueron seleccionadas previamente piezas multimodales del género narrativo en tres formatos diferentes: la historieta, el corto de vídeo y el libro álbum. Se 
buscó que abordaran temáticas controversiales para potenciar el intercambio verbal y la discusión entre los estudiantes. Los ejes temáticos de los textos elegidos giraron en torno a las dimensiones ética, estética y política, en las que entraron en juego categorías como la diversidad, el medio ambiente y la incidencia de las tecnologías en la vida del ser humano.

Las sesiones de intervención estuvieron integradas por tres momentos: actividades de apertura, actividades de desarrollo y actividades de cierre. El primero de ellos fue considerado como un espacio de tiempo para indagar los conocimientos previos y poner en contexto a los estudiantes en relación con el tema tratado en el texto. En el segundo se plantearon actividades que tendieron al análisis del texto multimodal propuesto para cada sesión, la identificación de la problemática abordada, el relato de los hechos ocurridos, el reconocimiento de la imagen y de los elementos verbales y no verbales a través de la mediación del docente con preguntas que promovieran la discusión. Finalmente, el tercer y último momento pretendió abrir espacios para plantear conclusiones, exponer ideas y plantear argumentos derivados de la temática central del texto.

Para cerrar, los diferentes elementos expuestos a lo largo del marco conceptual constituyen el enfoque teórico que orientó las líneas del proceso investigativo del cual da cuenta este artículo. Cada una de las categorías descritas, grosso modo, permiten hacerse una idea de la relevancia de la competencia discursiva como objeto de estudio y sustentan el marco de acción que permitió ponerla en diálogo con los textos multimodales, como una herramienta didáctica potente para ser empleada en el ámbito escolar.

\section{Resultados}

\section{Análisis descriptivo}

\section{Caracteristicas generales de la muestra}

Entre los aspectos analizados, se encuentra la distribución por sexo y edad registrados a continuación en la tabla 1. En cuanto al sexo, se aprecia una diferencia notable con respecto a la población matriculada en este nivel educativo en las instituciones oficiales de Antioquia. Para el caso del grupo de estudio, el $23 \%$ corresponde a niñas, cuando en el departamento en mención equivale al 48,5\%, es decir, el doble del observado en la muestra. Por otra parte, casi la totalidad de los estudiantes tenía siete años, pero también se registró un caso con ocho años. Estos resultados son consistentes con la edad en la que los estudiantes cursan este grado. 
Tabla 1. Clasificación de los estudiantes de segundo grado, según sexo y edad.

Medellín, Colombia, 2019

\begin{tabular}{ccccc}
\hline Variable & Categorías & Cantidad & $\%$ & $n$ \\
\hline \multirow{2}{*}{ Sexo } & Masculino & 5 & 77 & 8 \\
& Femenino & 3 & 23 & \\
\hline \multirow{2}{*}{ Edad } & Siete años & 7 & 87,5 & 8 \\
& Ocho años & 1 & 12,5 & \\
\hline
\end{tabular}

Fuente: elaboración propia a partir del programa SPSS.

\section{Características del entorno familiar}

Con el propósito de reconocer el medio en el cual se encuentran inmersos los estudiantes y las personas con las cuales interactúan a diario, se indagó acerca de elementos que pueden servir de punto de partida para caracterizar el componente sociodemográfico. En la tabla 2 se describen las particularidades de las familias. Con relación al nivel educativo, se puede afirmar que la mayoría de los padres alcanza el bachillerato $(62,5 \%)$ y ninguno ha realizado estudios superiores al tecnológico, lo que indica que el entorno familiar en el que se desenvuelven los estudiantes se caracteriza por no tener altos niveles de formación. Con respecto al número de hermanos, se observa que la mitad de los de los estudiantes son hijos únicos y, adicionalmente, todos comparten la mayor parte del tiempo con la mamá o los abuelos, pero predomina la mamá en un $62,5 \%$.

Tabla 2. Características del entorno familiar de los estudiantes de segundo grado.

Medellín, Colombia, 2019

\begin{tabular}{llccc}
\hline \multicolumn{1}{c}{ Variable } & \multicolumn{1}{c}{ Categorías } & Cantidad & $\%$ & $n$ \\
\hline \multirow{4}{*}{ Nivel educativo de los padres } & Primaria & 2 & 25,0 & 8 \\
& Bachillerato & 5 & 62,5 & \\
& Tecnológico & 1 & 12,5 & \\
\hline \multirow{2}{*}{ Número de hermanos } & Universitario & 0 & -- & \\
& Cero hermanos & 4 & 50,0 & 8 \\
& Un hermano & 3 & 37,5 & \\
\multirow{2}{*}{ Lugar que ocupa entre los hermanos } & Dos hermanos & 1 & 12,5 & \\
& Primero & 4 & 50,0 & 8 \\
& Segundo & 3 & 37,5 & \\
\multirow{2}{*}{ Persona con quien comparte la mayor parte del tiempo } & Mamá & 1 & 12,5 & \\
\hline & Tercero & 5 & 62,5 & 8 \\
& Abuelos & 3 & 37,5 & \\
\hline
\end{tabular}

Fuente: elaboración propia a partir del programa SPSS. 


\section{Actividades diferentes a las escolares}

Se entienden como actividades diferentes a las escolares las que los estudiantes desarrollan fuera del horario estudiantil establecido. Estas actividades pueden ser complementarias o de tiempo libre. Las primeras corresponden a aquellas que son dirigidas por una entidad externa, permiten a los estudiantes hacer uso de espacios diferentes en los que se fortalece alguna habilidad en particular y a las cuales los estudiantes asisten con regularidad. Las segundas hacen referencia al tiempo de ocio y libre esparcimiento. Desde el presente estudio se indagó acerca de estos aspectos para identificar las acciones que desarrollan con mayor frecuencia los estudiantes y determinar si pueden representar alguna incidencia en el desarrollo de la competencia discursiva.

\section{Actividades complementarias}

En la tabla 3 se registra que cinco de cada ocho estudiantes (62,5\%) asiste a actividades deportivas y que ninguno participa en actividades dirigidas por una biblioteca. Este hecho llama especialmente la atención, pues en el contexto en el que viven cuentan con acceso libre a dos bibliotecas en las que se desarrollan múltiples programas para favorecer la promoción de hábitos lectores y la participación en eventos culturales.

Tabla 3. Actividades complementarias que desarrollan fuera de la institución los estudiantes de segundo grado. Medellín, Colombia, 2019

\begin{tabular}{lccc}
\hline \multicolumn{1}{c}{ Actividades complementarias } & Cantidad & $\%$ & $n$ (total) \\
\hline Club de lectura & 0 & -- & 8 \\
Actividades culturales en la biblioteca & 0 & -- & \\
Escuela de música & 1 & 12,5 & \\
Actividades deportivas & 5 & 62,5 & \\
Ninguna & 2 & 25,0 & \\
\hline
\end{tabular}

Fuente: elaboración propia a partir del programa SPSS.

\section{Actividades que realizan en el tiempo libre}

En la tabla 4 se resumen las actividades que los estudiantes suelen hacer en su tiempo libre. En ella se aprecia que invierten mayor tiempo en ver televisión $(87,5 \%)$, seguido de la lectura y el dibujo (75 \%) y solo uno de cada ocho (12,5\%) usa el computador para consultar información. Dentro de estas actividades la que podría contribuir mayormente al desarrollo de la competencia discursiva es la lectura, ya que a través de ella pueden ampliar el vocabulario, además de fortalecer la capacidad para plantear ideas acerca de diferentes temas. 
Tabla 4. Actividades que realizan los estudiantes en su tiempo libre los estudiantes de segundo grado. Medellín, Colombia, 2019

\begin{tabular}{lccccc}
\hline \multirow{2}{*}{ Actividades } & \multicolumn{4}{c}{ Categoría } \\
\cline { 2 - 5 } & \multicolumn{3}{c}{ Sí } & \multicolumn{3}{c}{ No } \\
\cline { 2 - 5 } & Cantidad & $\%$ & Cantidad & $\%$ \\
\hline Jugar con sus amigos & 5 & 62,5 & 3 & 37,5 \\
Ver televisión & 7 & 87,5 & 1 & 12,5 \\
Leer & 6 & 75,0 & 2 & 25,0 \\
Escribir & 3 & 37,5 & 5 & 32,5 \\
Dibujar & 6 & 75,0 & 2 & 25,0 \\
Jugar videojuegos & 3 & 37,5 & 5 & 62,5 \\
Consultar información en el computador & 1 & 12,5 & 7 & 87,5 \\
\hline
\end{tabular}

Fuente: elaboración propia a partir del programa SPSS.

\section{Tiempo que invierten para conversar en familia y temas de conversación}

Entre otros aspectos considerados para el abordaje de la investigación, se encuentran aquellos que están relacionados con las posibilidades de diálogo generadas en el entorno familiar y la cantidad de tiempo que tienen para entablar conversaciones. Además, se pretendió reconocer los temas alrededor de los cuales hablan como una posibilidad para comprender si en este ambiente cercano los estudiantes cuentan con alguien a quien puedan relatar sus experiencias. En la tabla 5 se exponen los resultados al respecto. De acuerdo con estos, el $87,5 \%$ de los estudiantes interactúa con sus familias por un tiempo superior a las dos horas, situación que puede favorecer las posibilidades de expresión de ideas cotidianamente.

Tabla 5. Tiempo que los estudiantes de segundo grado dedican para conversar en familia. Medellín, Colombia, 2019

\begin{tabular}{lcc}
\hline \multicolumn{1}{c}{ Tiempo que dedican a conversar en familia } & Cantidad & $\%$ \\
\hline Una hora al día & 1 & 12,5 \\
De dos a cuatro horas al día & 4 & 50,0 \\
Más de cuatro horas al día & 3 & 37,5 \\
\hline
\end{tabular}

Fuente: elaboración propia a partir del programa SPSS.

Los espacios de conversación que se propicien en el ambiente familiar son relevantes para el objeto de estudio, ya que permiten identificar cómo es el contexto en el que se encuentran inmersos los estudiantes y las posibilidades 
que existen para intercambiar ideas con los miembros de su familia en torno a múltiples situaciones a las cuales se pueden enfrentar. Para tal efecto, en la tabla 6 se resumen los temas sobre los cuales gira la conversación familiar, lo que revela que hay mayor coincidencia (87,5\%) en lo relacionado con las situaciones que se presentan en sus hogares; el tema de menor conversación $(62,5$ $\%)$ es acerca de los libros que los estudiantes leen. Aunque este último se encuentra por encima de la mitad del grupo de estudio, entre las actividades que hacen en el tiempo libre se identifica que no suelen comentar con sus padres los libros que leen.

Tabla 6. Temas de conversación en familia, estudiantes de segundo grado.

Medellín, Colombia, 2019

\begin{tabular}{lccccc}
\hline \multirow{2}{*}{\multicolumn{1}{c}{ Temas de conversación }} & \multicolumn{4}{c}{ Categoría } \\
\cline { 2 - 5 } & \multicolumn{3}{c}{ Sí } & \multicolumn{3}{c}{ No } \\
\cline { 2 - 5 } & Cantidad & $\%$ & Cantidad & $\%$ \\
\hline Lo que sucedió en el colegio & 6 & 75,0 & 2 & 25,0 \\
Las situaciones que se presentan en casa & 7 & 87,5 & 1 & 12,5 \\
Los libros que lee & 5 & 62,5 & 3 & 37,5 \\
Los miedos y experiencias & 6 & 75,0 & 2 & 25,0 \\
\hline
\end{tabular}

Fuente: elaboración propia a partir del programa SPSS.

\section{Dimensiones de la competencia discursiva}

La competencia discursiva se encuentra integrada por tres dimensiones que corresponden a los procedimientos operativos del discurso propuestos por Charaudeau (2002). Para medir cada dimensión, se redactaron cuatro ítems derivados de la definición conceptual correspondiente y se registró la frecuencia de aparición del intercambio comunicativo. Los datos se extrajeron de los videos correspondientes a las cuatro sesiones, tanto en el pretest como en el postest. Como ya se indicó, una observadora independiente que funcionó como intercodificadora asignó los puntajes a cada participante, según criterios establecidos. Así, si no se produjo el intercambio verbal, se calificó con cero puntos (nunca); si se observó la conducta en una o dos de las sesiones, el puntaje correspondiente fue de un punto, equivalente a algunas veces. Por último, se ponderaron con dos puntos los casos en que se registró la conducta en al menos tres sesiones. La suma de las ponderaciones produjo un puntaje que oscila teóricamente entre cero y ocho puntos para cada dimensión y entre cero y veinticuatro puntos para la competencia discursiva total. A partir de ese puntaje, se calcularon promedios para describir y resumir la información obtenida. La tabla 7 resume los estadísticos descriptivos de la competencia discursiva pretest-postest, total y por 
dimensiones. Los resultados se analizan de manera separada por dimensiones. En general, los promedios en el postest fueron mayores a los registrados en el pretest.

Tabla 7. Estadísticos descriptivos pretest-postest de la competencia discursiva total y por dimensiones de los estudiantes de segundo grado. Medellín, Colombia, 2019

\begin{tabular}{lccccccc}
\hline \multirow{2}{*}{ Dimensión } & Medición & Promedio & Desviación típica & \multicolumn{3}{c}{ Puntaje } & \\
\cline { 6 - 7 } & & & & & Mínimo & Máximo & \\
\hline \multirow{2}{*}{ Dimensión enunciativa } & Pre & 6,25 & 1,58 & 4 & 8 & 8 \\
& Pos & 7,50 & 1,06 & 5 & 8 & 8 \\
\hline \multirow{2}{*}{ Dimensión descriptiva narrativan } & Pre & 4,50 & 2,73 & 1 & 8 & 8 \\
& Pos & 6,75 & 1,75 & 4 & 8 & 8 \\
\hline \multirow{2}{*}{ Dimensión argumentativa } & Pre & 2,38 & 1,85 & 0 & 4 & 8 \\
& Pos & 6,12 & 2,03 & 4 & 8 & 8 \\
\hline \multirow{2}{*}{ Competencia discursivan } & Pre & 13,30 & 5,61 & 5 & 20 & 8 \\
& Pos & 20,30 & 4,43 & 13 & 24 & 8 \\
\hline
\end{tabular}

Fuente: elaboración propia a partir del programa SPSS.

La dimensión enunciativa hace alusión a la actitud que asume el hablante frente a lo que expresa él mismo y su interlocutor, así como a su actuación acorde con las reglas sociales que rigen las prácticas discursivas según el género en el que están emergiendo. En la tabla 7 se puede identificar que, al inicio de la investigación, en esta dimensión los puntajes oscilaron entre cuatro y ocho puntos, es decir, los estudiantes alcanzaron el máximo puntaje posible, pero ninguno obtuvo el mínimo teórico (cero puntos), lo cual indica que se produjo al menos un intercambio verbal. El promedio pretest fue de 6,25 $\pm 1,58$ puntos, el cual supera en más de dos puntos la media teórica de cuatri puntos. Luego de la aplicación de la estrategia, se registró un aumento de más de un punto en esta dimensión (7,5 \pm 1,06 puntos). La desviación típica, indicador de la variabilidad de las respuestas, es menor en el postest, es decir, los puntajes están más próximos a la media.

Las respuestas al conjunto de ítems de la dimensión enunciativa revelan que la principal dificultad de estos estudiantes al inicio de la investigación radicaba en el rol que asumían como enunciadores o enunciatarios en los diferentes intercambios verbales. Luego de ser tratados con la estrategia, los estudiantes cambiaron su actitud de escucha y respetaron los turnos conversacionales. Se puede afirmar que la estrategia potenció los roles asumidos por los participantes en las diversas situaciones de comunicación, en los cuales inicialmente mostraban fortalezas y luego de la intervención se incrementaron con nuevas 
habilidades, como escuchar y complementar la opinión de su interlocutor. Así, asumieron, además, un rol activo como interlocutores en los procesos discursivos.

En segundo lugar, el puntaje promedio en la dimensión descriptiva-narrativa antes de la estrategia fue de 4,5 $\pm 2,73$ puntos, siendo los valores mínimo y máximo de uno y ocho puntos, respectivamente. Este promedio obtenido en la muestra es ligeramente superior a la media teórica (cuatro puntos). Tal como se esperaba, en el postest se produjo un incremento en el promedio de esta, al ubicarse dos puntos por encima del valor registrado en el pretest $6,75 \pm 1,75$ puntos). Además, disminuyó el valor de la desviación típica, es decir, los puntajes en el postest están más cercanos o concentrados alrededor de la media.

En tercer lugar, los puntajes pretest en la dimensión argumentativa oscilaron empíricamente entre cero y cuatro puntos. Ninguno de los estudiantes alcanzó el máximo teórico (ocho puntos) y la media del puntaje de los estudiantes $(2,38 \pm$ 1,85 puntos) está por debajo de la media teórica, lo cual indica que el desempeño en esta dimensión fue bajo. En el postest, el promedio registró un incremento de 2,57 puntos en relación con el pretest, al ubicarse en 6,12 $\pm 2,03$ puntos. Vale la pena destacar que se produjo un leve incremento en la variabilidad de los puntajes en el postest, contrario a lo observado en las dimensiones enunciativa y descriptiva-narrativa.

Para resumir las tres dimensiones descritas, se calculó el puntaje de la competencia discursiva de los estudiantes el cual, en la práctica para el pretest, osciló entre cinco y veinte puntos, con un promedio de 13,3 $\pm 5,62$, siendo esta media ligeramente superior a la media teórica (doce puntos). Al comparar el promedio obtenido por los estudiantes en el postest con el promedio pretest se observa que se produjo un incremento de siete puntos (20,3 $\pm 4,43$ puntos), es decir, un mejor desempeño en las habilidades discursivas luego de que los estudiantes participaron en la estrategia didáctica. Es oportuno resaltar que siete puntos en un rango de cero a veinticuatro puntos equivalen a un aumento del $29 \%$.

Si se comparan las tres dimensiones de la competencia discursiva, se destaca que en la dimensión argumentativa se registraron los mayores cambios, en la cual se produjo un ascenso de alrededor de cuatro puntos después de implementada la estrategia. No obstante, en todas las dimensiones aumentaron los puntajes postest con respecto a la puntuación inicial, lo que hace pensar que la estrategia basada en textos multimodales generó cambios en las habilidades discursivas que posteriormente se determinarían si son estadísticamente significativos, es decir, si la ventaja observada en el postest es producto o no del tratamiento experimental. 


\section{Análisis inferencial}

\section{Comparación de medias pretest-postest de las dimensiones de competencia discursiva}

Dado que las variables dependientes del estudio son cuantitativas por ser una escala aditiva obtenida de la suma de las ponderaciones de los ítems que forman las dimensiones (puntaje de las dimensiones enunciativa, descriptiva-narrativa y argumentativa), se midieron en dos tiempos los mismos sujetos, porque se deseaba conocer si había diferencias significativas en el puntaje promedio de cada dimensión. Se empleó la prueba $t$ de Student para muestras relacionadas a un nivel de significación del $5 \%(\alpha=0,05)$. Se debe mencionar que se eligió esta prueba estadística porque está diseñada especialmente para muestras pequeñas, es decir, menos de treinta sujetos (Pérez Juste et al., 2009). Se sometió a contraste la siguiente hipótesis nula, que se muestra en la ecuación 1:

Ecuación 1. Contraste de hipótesis nula

$$
\begin{aligned}
& \mathrm{H}_{0}: \mu_{1}=\mu \\
& \mathrm{H}_{1}: \mu_{1} \neq \mu_{2}
\end{aligned}
$$

Donde $\mu_{1}$ es el puntaje promedio de la dimensión (enunciativa; descriptivanarrativa; argumentativa) antes de la implementación de la estrategia didáctica basada en textos multimodales.

$\mu 2$ es el puntaje promedio de la dimensión (enunciativa; descriptiva-narrativa; argumentativa) después de la implementación de la estrategia didáctica basada en textos multimodales.

La tabla 8 contiene tanto los estadísticos descriptivos como los resultados de la prueba $t$ para muestras relacionadas. En dicha tabla se puede apreciar la diferencia promedio entre el pretest y el postest de las tres dimensiones de la competencia discursiva.

En primer lugar, se observa que el puntaje promedio de la dimensión enunciativa antes de la implementación de la estrategia (6,25 $\pm 1,58$ puntos) es menor al promedio de la dimensión enunciativa después de la implementación (7,5 $\pm 1,07$ puntos). La variabilidad es similar en ambas mediciones, dado que los valores de la desviación típica son semejantes. De igual modo, se aprecia el valor de la diferencia promedio antes y después de la estrategia, que se obtuvo al restar dichos promedios, cuyo valor es de 1,25 puntos, es decir, luego de la estrategia los estudiantes aumentaron en promedio sus puntajes en un poco más de un punto. El valor de probabilidad asociado a la prueba $t(\mathrm{p}=0,038)$ es menor que el nivel de significación de la investigación $(\alpha=0,05)$. Por tanto, se rechaza la hipótesis nula de igualdad de medias y se concluye que existen 
diferencias estadísticamente significativas en los puntajes promedio de la dimensión enunciativa antes y después de la implementación de la estrategia. Puede decirse, por tanto, que después de finalizada la intervención, el hecho de que el promedio del puntaje de la dimensión enunciativa haya aumentado en 1,25 $\pm 1,39$ puntos, se debe a la estrategia didáctica (diferencia de medias pretest-postest $=1,25 ; t=2,546 ; p=0,038$ ). La verdadera diferencia promedio se ubica entre 0,089 y 2,411 puntos a un nivel de confianza del $95 \%$.

Asimismo, resulta interesante poner de manifiesto que, luego de la intervención con la estrategia didáctica, los resultados para la dimensión enunciativa arrojaron otras transformaciones positivas, esta vez relacionadas con la capacidad de escucha de los participantes y su actitud como enunciadores frente a los diferentes actos de habla. Al finalizar el proceso, los estudiantes entrelazaron el recuento inicial del compañero con el propio, recurrieron al vocabulario empleado en los textos multimodales para expresar sus ideas. Además, en sus intervenciones consideraron tanto a su interlocutor como al contexto en que se producía la situación de comunicación para expresar sus ideas.

Tabla 8. Prueba t para muestras relacionadas del promedio de las dimensiones de competencia discursiva de estudiantes de segundo grado. Medellín, Colombia, 2019

\begin{tabular}{|c|c|c|c|c|c|c|c|c|}
\hline \multirow[t]{2}{*}{ Dimensiones } & & \multirow[t]{2}{*}{ Promedio } & \multirow{2}{*}{$\begin{array}{l}\text { Diferencia } \\
\text { promedio }\end{array}$} & \multirow{2}{*}{$\begin{array}{c}\text { Desviación } \\
\text { típica }\end{array}$} & \multicolumn{2}{|c|}{$\begin{array}{c}\text { Intervalo de } \\
\text { confianza } 95 \%\end{array}$} & \multirow[t]{2}{*}{$t$} & \multirow[t]{2}{*}{$p$} \\
\hline & & & & & Inferior & Superior & & \\
\hline \multirow{2}{*}{ Enunciativa } & Pre & 6,25 & \multirow{2}{*}{1,25} & \multirow{2}{*}{1,39} & \multirow{2}{*}{0,089} & \multirow{2}{*}{2,411} & \multirow{2}{*}{2,546} & \multirow{2}{*}{$0,038^{*}$} \\
\hline & Pos & 7,50 & & & & & & \\
\hline \multirow{2}{*}{ Descriptiva } & Pre & 4,50 & \multirow{2}{*}{2,25} & \multirow{2}{*}{1,49} & \multirow{2}{*}{1,006} & \multirow{2}{*}{3,494} & \multirow{2}{*}{4,277} & \multirow{2}{*}{$0,004^{* *}$} \\
\hline & Pos & 6,75 & & & & & & \\
\hline \multirow{2}{*}{ Argumentativa } & Pre & 2,38 & \multirow{2}{*}{3,75} & \multirow{2}{*}{1,67} & \multirow{2}{*}{2,355} & \multirow{2}{*}{5,145} & \multirow{2}{*}{6,355} & \multirow{2}{*}{$0,000^{* *}$} \\
\hline & Pos & 6,12 & & & & & & \\
\hline
\end{tabular}

* Significativo $\alpha<0,05$

** Significativo $\alpha<0,01$

Fuente: elaboración propia a partir del programa SPSS.

En segundo lugar, los puntajes promedios de la dimensión descriptivanarrativa antes y después de la intervención son respectivamente 4,50 $\pm 2,73$ puntos y $6,75 \pm 1,75$ puntos. Se aprecia, entonces, un aumento superior a los dos puntos en el postest de esta dimensión. Dado que el valor $p$ del estadístico $t$ es menor que 0,05 , se rechaza la hipótesis nula y se concluye que sí existen diferencias significativas desde el punto de vista estadístico entre los promedios en las dos mediciones, lo que permite afirmar que la implementación de la estrategia didáctica ejerció una influencia altamente significativa en dicha di- 
mensión (diferencia pretest-postest $=1,25 ; t=4,277 ; p=0,004$ ). Un intervalo de confianza al $95 \%$ para la diferencia del puntaje promedio antes y después de la intervención, revela que la verdadera diferencia pretest-postest está ubicada en algún punto del intervalo comprendido entre 1,006 y 3,494 puntos.

En el caso de la dimensión descriptiva, el grupo experimental presentó cambios relacionados con la fluidez en el relato de los hechos acontecidos en los textos, el reconocimiento de la trama discursiva, así como el empleo de recursos narrativos y descriptivos soportados en las imágenes que integraban las piezas multimodales seleccionadas.

En tercer lugar, la dimensión argumentativa refleja que hay diferencias significativas entre el puntaje promedio antes de la estrategia (2,38 $\pm 1,85$ puntos) y después de aplicada (6,13 $\pm 2,03$ puntos), es decir, se produjo un incremento de 3,75 $\pm 1,669$ puntos siendo este aumento estadísticamente significativo, ya que el valor de probabilidad asociado al estadístico $t$ es menor que el nivel de significación elegido en la investigación (diferencia pretest-postest $=6,1$; $t=6,355 ; p=0,000$ ). A un nivel de confianza del $95 \%$, la verdadera diferencia de puntajes promedio pretest-postest se encuentra entre 2,355 y 5,145 puntos.

En esta dimensión se observaron las mayores transformaciones. En efecto, antes de la implementación de la estrategia didáctica basada en textos multimodales, los estudiantes no asumían posiciones frente a las intervenciones de sus compañeros o las problemáticas expuestas en los textos y planteaban sus proposiciones sin ningún tipo de argumento. Luego de la intervención, los participantes lograron justificar sus respuestas, expresaron su coincidencia o diferencia acerca de lo expuesto por sus pares, asumieron posiciones frente a los hechos ocurridos en los textos, explicaron su identificación o no con los personajes y dieron las razones que los llevaron a establecer esta relación.

\section{Comparación de medias de la competencia discursiva pretest postest}

Como se indicó en la metodología, las tres dimensiones analizadas conformaban la competencia discursiva, cuyos resultados oscilaban entre cero y veinticuatro puntos. La diferencia pretest-postest que se presenta en la tabla 9 muestra que los puntajes de los estudiantes aumentaron en 7,25 $\pm 2,66$ puntos luego de la aplicación de la estrategia didáctica. Los resultados de comparación de medias relacionadas con la prueba $t$ conllevan a rechazar la hipótesis de que las medias pretest-postest son iguales y se concluye que el uso de textos multimodales influyó de manera altamente significativa en la competencia discursiva (diferencia pretest-postest $=7,25 ; t=7,711 ; p=0,000$ ). 
Mary Luz Hoyos Hoyos, Gladys Lucía Acosta Valencia y Solbey Morillo Puente

Tabla 9. Prueba $t$ para muestras relacionadas de comparación de medias de la competencia discursiva pretest-postest, estudiantes de segundo grado. Medellín, Colombia, 2019

\begin{tabular}{|c|c|c|c|c|c|c|c|}
\hline \multirow{2}{*}{$\begin{array}{c}\text { Competencia } \\
\text { discursiva }\end{array}$} & \multirow{2}{*}{ Promedio } & \multirow{2}{*}{$\begin{array}{l}\text { Diferencia } \\
\text { promedio }\end{array}$} & \multirow{2}{*}{$\begin{array}{l}\text { Desviación } \\
\quad \text { típica }\end{array}$} & \multicolumn{2}{|c|}{ Intervalo de confianza $95 \%$} & \multirow{2}{*}{$t$} & \multirow{2}{*}{$p$} \\
\hline & & & & Inferior & Superior & & \\
\hline Pretest & 13,30 & \multirow{2}{*}{7,250} & \multirow{2}{*}{2,66} & \multirow{2}{*}{5,03} & \multirow{2}{*}{9,47} & \multirow{2}{*}{7,711} & \multirow{2}{*}{$0,000 * *$} \\
\hline Postest & 20,30 & & & & & & \\
\hline
\end{tabular}

* Significativo $\alpha<0,01$

Fuente: elaboración propia a partir del programa SPSS.

Los resultados obtenidos en la competencia discursiva dan cuenta de un avance importante en las habilidades comunicativas de los estudiantes intervenidos, quienes se posicionaron como participantes activos de las prácticas dialógicas propiciadas en el aula de clase mediante la utilización de diversos medios discursivos para interactuar en las situaciones de comunicación.

\section{Comparación de medias de la competencia discursiva pretest según sexo}

Se realizó esta prueba para verificar que los grupos formados a partir del sexo son equivalentes en su competencia discursiva, se sometió a contraste la hipótesis nula de igualdad de medias, que se expresa en la ecuación 2 :

Ecuación 2. Contraste de hipótesis nula de igualdad de medias

$$
\mathrm{H}_{0}: \mu_{1}=\mu_{2}
$$

$$
\mathrm{H}_{1}: \mu_{1} \neq \mu_{2}
$$

Donde $\mu_{1}$ es el promedio de la competencia discursiva pretest de los niños y

$\mu 2$ es el promedio de la competencia discursiva de las niñas.

Se empleó la prueba $t$ de Student para muestras independientes a un nivel de significación del $5 \%$, dado que la variable dependiente competencia discursiva es cuantitativa y se tienen dos grupos de sexo. Las medias muestrales de esta competencia revelan que los niños tienen un mayor promedio $(14,5 \pm 5,96$ puntos) que las niñas (11,7 $\pm 5,86$ puntos). Por otra parte, los resultados de la prueba $t$ indican que la diferencia promedio de los niños y de las niñas es de 2,33 puntos, lo cual indica que los primeros obtuvieron en promedio 2,33 puntos más en su competencia discursiva.

Se verificó el cumplimiento de los supuestos de homoscedasticidad mediante la prueba de Levene $(F=0,039 ; p=0,849)$ y de normalidad a través de la prueba 
de Kolmogorov-Smirnov $(K S=0,187 ; p=0,200)$. El valor de probabilidad asociado a la prueba $t(0,539)$ señala que no se debe rechazar la hipótesis nula $(\mathrm{p}=0,609$ $>0,05)$. En conclusión, la diferencia observada en las medias muestrales no es estadísticamente significativa. Esto quiere decir que los niños no difieren de las niñas de manera significativa en su competencia discursiva.

Se aplicó la misma prueba para contrastar las hipótesis de similitud de promedios de competencia discursiva entre los otros grupos de variables, y se identificó que no existen diferencias estadísticamente significativas en ninguno de los grupos. Como se evidencia en la tabla 10, el valor $p$ asociado a la diferencia de medias de la competencia discursiva para temas de conversación sobre lo que sucedió en el colegio, es mayor a 0,05 ( $p=0,228>0,05)$.

Tabla 10. Comparación de medias de competencia discursiva según las variables, estudiantes de segundo grado. Medellín, Colombia, 2019

\begin{tabular}{lccccccc}
\hline Variable independiente & Grupo & $n$ & $\begin{array}{c}\text { Promedio } \\
\text { competencia } \\
\text { discursiva }\end{array}$ & $\begin{array}{c}\text { Desviación } \\
\text { típica }\end{array}$ & $\begin{array}{c}\text { Diferencia } \\
\text { de medias }\end{array}$ & $\begin{array}{c}\text { Pruebat de } \\
\text { Student }\end{array}$ & $P$ \\
\hline Sexo & $\mathrm{M}$ & 5 & 14 & 5,95 & 2,33 & 0,539 & 0,609 \\
\cline { 2 - 9 } & $\mathrm{F}$ & 3 & 11,67 & 5,85 & & & \\
\hline $\begin{array}{l}\text { Temas de } \\
\text { conversación: lo que } \\
\text { sucedió en el colegio }\end{array}$ & No & 2 & 17,50 & 3,54 & & & \\
\cline { 2 - 9 } & Sí & 5 & 14,2 & 5,1 & 2,87 & 0,673 & 0,527 \\
\hline $\begin{array}{l}\text { Temas de } \\
\text { conversación: libros } \\
\text { que leen }\end{array}$ & No & 3 & 11,3 & 7,1 & & & \\
\hline
\end{tabular}

Fuente: elaboración propia a partir del programa SPSS.

\section{Conclusiones}

Una vez finalizado el proceso de implementación de la estrategia didáctica basada en textos multimodales con los estudiantes de segundo grado de una institución oficial de Antioquia, se puede llegar a las siguientes conclusiones:

Los resultados obtenidos luego de la intervención demuestran que la estrategia didáctica La competencia discursiva potenciada a través de los textos multimodales surtió el efecto esperado dando cumplimiento al propósito central de la investigación, puesto que logró fortalecer la competencia discursiva de los estudiantes. Lo anterior se reflejó en el mejoramiento de las habilidades discursivas de los participantes, el incremento de intercambios verbales, la expresión coherente de ideas con un hilo conductor y la presentación de argumentos para 
justificar sus opiniones con el propósito de persuadir a su interlocutor, lo que se refleja en los resultados estadísticos del postest.

Al concluir las sesiones se observó que los estudiantes habían avanzado en cada una de las dimensiones que integran la competencia en mención. Para el caso de la dimensión enunciativa, se percibió en ellos una actitud de escucha activa y la expresión de sus ideas en consonancia o disonancia con relación a los planteamientos de los demás. Con respecto a la dimensión descriptiva-narrativa, los participantes relataron hechos de manera coherente y emplearon un vocabulario fluido y variado. Además, enunciaron sus ideas teniendo en cuenta el contexto comunicativo. Finalmente, con relación a la dimensión argumentativa se puede decir que, en las interacciones generadas entre los participantes, se observaron intentos de persuasión al interlocutor y expresaron seguridad frente a las ideas que defendían a través de argumentos que las complementaban.

La dimensión que presentó un mayor incremento fue la argumentativa, porque antes de la intervención los estudiantes no justificaban sus ideas ni se preocupaban por rebatir los puntos de vista de sus compañeros. Al terminar el proceso, fue notoria la utilización de explicaciones y argumentos para que sus ideas fueran escuchadas y validadas por sus pares.

Cabe destacar que de los antecedentes consultados con relación a la competencia discursiva, prevalece el enfoque cualitativo, lo que permite deducir que esta competencia ha sido poco estudiada desde la perspectiva cuantitativa. Este aspecto se convierte en una característica diferenciadora del presente trabajo investigativo en virtud de que a través del análisis estadístico se caracterizaron aspectos que integran esta competencia mediante constructos teóricos para hacer mediciones que permitan constatar conductas propias del accionar discursivo de los sujetos.

Los estudios cualitativos consultados como antecedentes aluden a los componentes enunciativo, descriptivo-narrativo y argumentativo que integran la competencia discursiva, pero son pocos los que contemplan el concepto de manera sistémica y conjugan los tres aspectos en mención. Esto sugiere la necesidad de que se abra el campo de acción en el área investigativa para favorecer procesos enmarcados en el conjunto de habilidades implicadas al momento de potenciarla.

Los textos multimodales son un recurso rico en lenguaje verbal y no verbal, lo que permite desarrollar habilidades discursivas de los estudiantes, dado que apelan a la imagen, el paralenguaje, los enunciados escritos, entre otros recursos que permiten describir, narrar y dar explicaciones a partir de los 
acontecimientos que presentan. Además, las temáticas que tratan generan la reflexión y el diálogo entre los interlocutores.

El ámbito educativo no puede ser ajeno a las transformaciones sociales generadas a raíz del creciente auge de las tecnologías, lo que ha llevado a los sujetos a estar en contacto diario con textos diferentes a los monomodales. Por tanto, su papel es atender este asunto instaurando el texto multimodal como parte de la dinámica escolar, pues brinda herramientas necesarias para que los docentes configuren nuevas miradas y se acerquen a ellos con un lente crítico que les permita argumentar su posición frente a los distintos mensajes verbales y no verbales que reciben.

\section{Agradecimientos}

Este artículo es el resultado del trabajo de investigación realizado en la maestría en Educación de la Universidad de Medellín, cuyo título fue La competencia discursiva en estudiantes de segundo grado potenciada a través de una estrategia didáctica basada en textos multimodales, terminado en 2020.

\section{Referencias}

Adán, N. (2014). La expresión oral en el aula de Educación Primaria: una propuesta didáctica [tesis de pregrado, Universidad de Jaén, España]. Repositorio de Trabajos Académicos de la Universidad de Jaén. http://tauja.ujaen.es/handle/10953.1/1361

Arenas, G. y Moreno, D. (2014). Habilidades cognitivas en la escritura de textos multimodales. Estudio exploratorio con estudiantes en educación básica primaria [tesis de pregrado, Universidad de Antioquia, Colombia]. Repositorio digital. http://ayura.udea.edu.co:8080/jspui/handle/123456789/1188

Avendaño, F. (2007). Hablar en clase: habla el docente, habla el alumno. Anales de Educación Común, 3(6), 132-137.

Bajtín, M. (1982). El problema de los géneros discursivos. En M. Bajtín (coord.), Estética de la creación verbal (pp. 248-293). Siglo XXI Editores.

Bravo, L. (2015). Aplicación de lecturas dialógicas en educación infantil [tesis de pregrado, Universidad Jaume I, España]. Repositori Universitat Jaume I. http://repositori.uji.es/xmlui/handle/10234/163912

Cruz, R., Ordóñez, R., Román, S. y Pavón, F. (2016). Buenas prácticas que desarrollan la competencia mediática en entornos socioeducativos. Revista de Medios y Educación, 48, 97-113. http://www. redalyc.org/articulo.oa?id $=36843409008$

Chacón, A. (2015). La multimodalidad del cómic: una experiencia de lectura de imágenes en los primeros grados escolares. Enunciación, 20(2), 190-206. https://doi.org/10.14483/udistrital.jour. enunc.2015.2.a02

Charaudeau, P. (1983). Una teoría de los sujetos del lenguaje. Magazine Littéraire. (200), 53-67. http://ru.iis.sociales.unam.mx/jspui/bitstream/IIS/5436/2/03_charaudeau.pdf 
Mary Luz Hoyos Hoyos, Gladys Lucía Acosta Valencia y Solbey Morillo Puente

Charaudeau, P. (2001). De la competencia social de comunicación a las competencias discursivas. Revista Latinoamericana de Estudios del Discurso, (1), 1-25. http://www.patrick-charaudeau.com/ De-la-competencia-social-de.html

Charaudeau, P. (2002). Una concepción comunicativa del discurso. Discourse Studies, 4(3), 1-22. http:// www.patrick-charaudeau.com/A-communicative-conception-of.html

Estela, R. (2018). Modelo metadiscursivo para mejorar la competencia argumentativa oral en estudiantes de segundo ciclo de educación superior [tesis de maestría, Universidad Privada Antenor Orrego, Perú]. Repositorio de Tesis Upao. http://repositorio.upao.edu.pe/handle/upaorep/4224?mode=full

Feo, R. (2010). Orientaciones básicas para el diseño de estrategias didácticas. Tendencias Pedagógicas, 16, 220-236. https://revistas.uam.es/tendenciaspedagogicas/article/view/1951/2062

Gómez, M. (2017). El comportamiento lector en textos multimodales digitales en la básica primaria [tesis de maestría, Universidad de Antioquia, Colombia]. Repositorio digital UDEA. http://ayura.udea. edu.co:8080/jspui/handle/123456789/3217

Gutiérrez, R. (2013). La compleja tarea de evaluar la oralidad: una propuesta de evaluación auto/formativa. Enunciación, 18(1), 109 -117.

Haquin, M., González, D., Echeverría, E., Marín, C., Rodríguez, P. y Guajardo, V. (2013). Leer para aprender a partir de textos multimodales: los materiales escolares como mediadores semióticos. Revista de Estudios y Experiencias en Educación, 12(24), 77-91. http://www.redalyc.org/articulo. oa?id $=243129663005$

Keilwitz, M. y Correa, N. (2014). Prácticas de enseñanza de escritura multimodal mediadas por las TIC, como alternativa de expresión y comunicación en la educación básica primaria [tesis de pregrado, Universidad de Antioquia, Colombia]. Repositorio digital. http://ayura.udea.edu.co:8080/jspui/ handle/123456789/1119

Kress, Gunther y Van Leeuwen, Theo. (2001). Multimodal Discourse. The Modes and Media of Contemporary Communication. Arnold.

Lafontaine, L y Hébert, M. (2013). La enseñanza-evaluación integrada de la oralidad reflexiva en las situaciones de aprendizaje de lectura entre pares en la enseñanza primaria y secundaria. Enunciación. 18(1), 8-28. https://revistas.udistrital.edu.co/index.php/enunc/article/view/7450

Lemke, J. (1998). Multiplying meaning: visual and verbal semiotics in scientific text. Martin \& Veel. En J. Martin y R. Veel (eds.), Reading Science: critical and functional perspectives on discourses of science (pp. 87-113). Routledge.

Manghi, D. (2013). Géneros de la enseñanza escolar: configuraciones de significado en clases de historia y biología desde una perspectiva multimodal. Revista Signos, 46(82), 236-257. http://dx.doi. org/10.4067/S0718-09342013000200004

Martín, M. y Jiménez, A. (2013). Las tertulias literarias dialógicas, un desafío para la creatividad y la convivencia. Creatividad y Educación, (21), pp. 1-21. http://creatividadysociedad.com/wp-admin/ Art\%C3\%ADculos/21/8.\%20Las\%20tertulias\%20literarias\%20dialogicas\%2C\%20un\%20desafio\%20 para\%20la\%20creatividad\%20y\%20la\%20convivencia.pdf?_t=1576012009

Méndez, S. Urrea, G. y Ayala, M. (2016). Estrategias de enseñanza-aprendizaje para desarrollar competencias verbal y escrita en un aula de primaria multigrado. Revista de Investigación Educativa de la Escuela de Graduados en Educación. 6(12), 31-36. https://www.rieege.mx/index.php/rieege/ article/view/263/235 
Mendioroz, A. y Asiáin, A. (2017). Empleo de la fotografía para desarrollar la alfabetización multimodal. Revista de Didácticas Específicas, 16, 80-95. https://revistas.uam.es/didacticasespecificas/ article/view/6756/8363

Melo, A. (2014). Procesos de producción y comprensión de textos multimodales a través del libro álbum. Folios de Humanidades y Pedagogía, (2), 37-56. https://revistas.pedagogica.edu.co/index. $\mathrm{php} / \mathrm{FHP} /$ article/view/2555

Monsalve, M. (2013). Habilidades argumentativas en la producción de textos con características discursivas multimodales [tesis de maestría, Universidad de Antioquia, Colombia]. Repositorio digital. http://ayura.udea.edu.co:8080/jspui/handle/123456789/1907

Monsalve, M., Chaverra, D., y Bolívar, W. (2015). Caracterización y evaluación de la habilidad razonabilidad en la producción escrita de textos multimodales. Forma y Función, 28(2), 111-133. https://doi.org/10.15446/fyf.v28n2.53543

Montes, L. (2016). Para hablarte mejor. Una experiencia de abordaje de la oralidad desde la narración oral y los lenguajes multimodales. Revista Oralidades, 2(4), pp. 42-46. https://revistaoralidad-es. com/index.php/ro-es/article/view/52

Núñez, M. (2000). Un aspecto básico para la didáctica de la lengua oral: el papel del lenguaje en la comunicación didáctica. Lenguaje y Textos, 16, 155-172. https://ruc.udc.es/dspace/handle/2183/8130

Oliveira, J., Camacho, M. y Gisbert, M. (2014). Explorando la percepción de estudiantes y profesor sobre el libro de texto electrónico en educación primaria. Comunicar, (42), 87-95. http://dx.doi. org/10.3916/C42-2014-08

Oteíza, T. (2009). Diálogo entre textos e imágenes: análisis multimodal de textos escolares desde una perspectiva intertextual. Delta, 25, 664-667. http://www.scielo.br/pdf/delta/v25nspe/ a08v25nspe.pdf

Pedrosa, I., Suárez, J. y García, E. (2013). Evidencias sobre la validez de contenido: Avances teóricos y métodos para su estimación. Acción Psicológica, 10(2), 3-18. http://dx.doi.org/10.5944/ap.10.2.11820

Pérez Juste, R., García Llamas, J., Gil Pascual, J. y Galán González, A. (2009). Estadística aplicada a la educación. Pearson Educación, S.A.

Piñeros, F. y Sepúlveda, Y. (2017). El cómic: un pretexto para mejorar la lectura intertextual con estudiantes de grado séptimo [tesis de Maestría, Universidad Distrital Francisco José de Caldas, Colombia]. http://repository.udistrital.edu.co/handle/11349/6994

Rodríguez, M. (1995). Hablar en la escuela: ¿para qué? ¿cómo? Lectura y Vida, 16(3), 1-12.

Rojas, E. (2013). La lectura de textos multimodales en el contexto de proyectos de aprendizaje en la Escuela Primaria [tesis doctoral, Universidad Autónoma de Barcelona, España]. Dipòsit digital de documents de la UAB. https://ddd.uab.cat/record/118665?ln=ca

Serrano, R. González, E. y Olivares, M. (2017). Estrategias y modalidades de argumentación oral en las asambleas de Educación Infantil. Un estudio multicaso. Revista Complutense de Educación. 28(2), pp. 445-462. http://dx.doi.org/10.5209/rev_RCED.2017.v28.n2.49439

Vásquez, F. (2011). La didáctica de la oralidad: experiencia, conocimiento y creatividad. Enunciación, 16(1), pp. 151-160. 